University of Nebraska - Lincoln

DigitalCommons@University of Nebraska - Lincoln

October 1981

\title{
Piezomodulation spectroscopy of molecular crystals. IV. The first singlet systems of TCNQ and BDP
}

\author{
J. Merski \\ University of Nebraska - Lincoln \\ Craig J. Eckhardt \\ University of Nebraska - Lincoln, ceckhardt1@unl.edu
}

Follow this and additional works at: https://digitalcommons.unl.edu/chemistryeckhardt

Part of the Chemistry Commons

Merski, J. and Eckhardt, Craig J., "Piezomodulation spectroscopy of molecular crystals. IV. The first singlet systems of TCNQ and BDP" (1981). Craig J. Eckhardt Publications. 33.

https://digitalcommons.unl.edu/chemistryeckhardt/33

This Article is brought to you for free and open access by the Published Research - Department of Chemistry at DigitalCommons@University of Nebraska - Lincoln. It has been accepted for inclusion in Craig J. Eckhardt Publications by an authorized administrator of DigitalCommons@University of Nebraska - Lincoln. 


\title{
Piezomodulation spectroscopy of molecular crystals. ${ }^{\text {a) }}$ IV. The first singlet systems of TCNQ and BDP')
}

\author{
J. Merski ${ }^{\mathrm{c}}$ and C. J. Eckhardt ${ }^{\mathrm{d})}$ \\ Department of Chemistry, University of Nebraska, Lincoln, Nebraska 68588 \\ (Received 9 October 1980; accepted 3 March 1981)
}

The polarized modulated piezoreflection spectra of the first singlet system of TCNQ and BDP are reported. Kramers-Kronig transformations are obtained and polariton energy strain shifts are determined. In contrast to systems with negligible to intermediate coupling, the piezomodulation spectrum for the mixed transverselongitudinal polaritons show strong deviations from the logarithmic frequency derivative of the corresponding reflection spectra. Progressions in the piezomodulation spectra in the energy region of the stop band are related to multiparticle states. Piezomodulation spectroscopy is shown to exhibit greatest deviation from derivative behavior when solid state effects become extensive.

\section{INTRODUCTION}

Previous studies in this series have considered the effect of lattice stain on the exciton spectra of single crystals of pyromellitic dianhydride-anthracene complex (PMDA-A) and anthracene. The piezomodulation spectra observed for these noncoupled and weak to intermediately coupled systems are simple or slightly perturbed derivatives with respect to the transition energy. The exciton (or polariton) strain coupling in the p-tetracyanoquinodimethane (TCNQ) and 1,5-bis(dimethylamino)pentamethinium perchlorate (BDP) systems which exhibit crystal spectra characteristic of strong coupling are reported here.

The molecular transition oscillator strengths for the first singlet transitions of TCNQ and BDP are greater than unity. ${ }^{1,2}$ These, together with efficient molecular packing in the lattice, result in crystal transitions which exhibit unusually wide bandwidths. These polariton stop bandwidths exceed the single molecule Franck-Condon bandwidths. The Simpson-Peterson ${ }^{3}$ coupling criterion is

$$
\theta=\frac{4 \pi \mu^{2}}{\epsilon_{0} v_{0}}>\Delta \text {, }
$$

where $\theta$ represents the Coulomb exciton bandwidth of the electronic excitation in the crystal and $\Delta$ corresponds to the energy interval spanned by the vibronic progression in the single molecule spectrum. For $\theta>\Delta$, the crystal absorption spectrum, which typically bears little resemblance to that of the single molecule, is determined by the relative magnitudes of and interplay between the exciton-exciton and exciton-photon coupling. The latter is particularly important for crystal faces upon which there is a large projection of an intense molecular transition. Since this obtains, the polariton stopping band results in a broad band crystal

\footnotetext{
a) Research supported by the Solid State Chemistry Program, Division of Materials Research of the National Science Foundation (DMR 79-08-759).

b) Part I of the series is J. Merski and C. J. Eckhardt, J. Chem. Phys. 75, 3691 (1981); part II is 75, 3705 (1981); part III is 75, 3719 (1981).

c) Part of a dissertation submitted to the University of Nebraska in partial requirement for the $\mathrm{Ph} . \mathrm{D}$. degree.

Dohn Simon Guggenheim Fellow, 1979-80.
}

reflection spectrum wherein the magnitude of the reflectivity $R$ approaches $100 \%$. Such a phenomenon may be characterized as quasimetallic reflection from an organic crystal. Using terminology introduced in Ref. 1, TCNQ and BDP are quasimetallic reflectors at optical frequencies. ${ }^{1,2}$ Spectra of the latter also exhibit features attributable to both manifolds of single particle vibronic exciton states and multiparticle vibronic-vibrational resonances which become spectroscopically observable by stealing intensity from the single particle states. The energy spacings and either hyper- or hypochromicity of the observed vibronic structure are rather sensitive functions of the coupling among the closely spaced vibronic levels. ${ }^{4}$ This imparts a degree of complexity to the exciton-polariton spectra of TCNQ and BDP which is not observed in the spectra of the very weak and intermediate coupling systems PMDA-A and anthracene.

The semiclassical dipole theory of single particle vibronic polariton states for a model isotropic crystal comprised of anisotropic molecules is adequate to describe the principal features of the TCNQ and BDP spectra. $4,5,8$ Treatments of vibronic coupling which include the influence of multiparticle states on the observed spectra of strong coupling systems have been given by Philpott $^{7}$ and Johnson and Simpson. ${ }^{6}$

It is convenient to partition a consideration of crystal interactions in strong coupling systems into those dealing with exciton-photon and vibronic coupling. The dispersion relations for a manifold of single particle vibronic polaritons may be obtained from the solutions of Eq. (3.3) of paper I:

$\tilde{\epsilon}(\omega, \mathbf{k})=\tilde{n}^{2}(\omega, \mathbf{k})=\frac{c^{2} \tilde{k}^{2}}{\omega^{2}}=\epsilon_{0}\left[1+\sum_{u} \frac{\omega_{0}^{2} F_{u}(\hat{\mu} \cdot \hat{\mathbf{e}})^{2}}{\Omega_{u}^{2}-\omega^{2}+i \gamma_{u} \omega}\right]$.

The arguments are defined in paper $I$. The polariton dispersion curves show (paper I, Fig. 3) that the two branches defining a particular polariton dispersion relation asymptotically approach the assumed dispersionless Coulomb exciton energies at $k \rightarrow \infty$ and at $k=0$. The interval spanned by the $\mathbf{k}-\infty$ to $\mathbf{k}=0$ polariton energies defines the reflection stop band over which $R=100 \%$ in the absence of damping.

Implicit in Eq. (1.1) and Eqs. (4.35) and (4.36) of paper $I$ is the retarded wave sum of transition dipole- 
dipole interactions which govern the energy separation between the $k \rightarrow \infty$ and $k=0$ polaritons derived from the $u$ th molecular transition. This retarded dipole sum may be written as

$\mathbf{I}(\omega, \mathbf{k})=i(\mathbf{k})+\frac{4 \pi \mu^{2}}{\epsilon_{0} v_{0}}(\hat{\mu} \cdot \hat{\mathbf{k}})^{2}+\frac{4 \pi \mu^{2}}{\epsilon_{0} v_{0}} \frac{(\hat{\mu} \cdot \hat{\mathbf{e}})^{2}}{1-n^{2}}$,

where $\hat{\mu}, \hat{\mathbf{k}}$, and $\hat{\mathbf{e}}$ are unit vectors defining the polarization of the transition, the photon wave vector, and photon polarization, respectively. The first two terms on the right hand side of Eq. (1.2) are the nonretarded analytic and macroscopic dipole interactions, respectively. The remaining term corresponds to macroscopic retarded interactions devoid of contributions from photon Umklapp processes. Evaluation of Eq. (1.2) for transverse polaritons $(\hat{\mu} \cdot \hat{\mathbf{k}}=0)$ corresponding to $\mathrm{k} \rightarrow \infty$ and $k=0$ limiting values reveals that the stop bandwidth may be approximated as $\left(4 \pi \mu^{2} / \epsilon_{0} v_{0}\right)(\hat{\mu} \cdot \hat{e})^{2}$, which reduces to the Simpson-Peterson result when $\hat{\mu} \cdot \hat{\mathbf{e}}=1$. The TCNQ (010) and BDP (010) spectra arise from the excitation of transverse polaritons for which the preceding obtains. In contrast, the a polarized TCNQ (001) face spectrum is that of a mixed transverse-longitudinal polariton for which $\hat{\mu} \cdot \hat{\mathbf{k}} \neq 0$.

The foregoing illustrates the functional dependence of the polariton bandwidth on macroscopic interactions which themselves depend on the unit cell volume $v_{0}$. This implies that piezomodulation spectra of strongly coupled systems may be expected to yield evidence for strain-induced deformations of polariton bands. Fur thermore, the mixed transverse-longitudinal polariton observable for the a polarization of TCNQ (001) should show a piezomodulation response different from that of the TCNQ (010) face transverse polariton which is devoid of a longitudinal field.

Large coupling energies lead to the formation of a $\mathrm{col}$ lective vibronic exciton which is characterized by an effective Franck-Condon factor of unity. Additional hypochromic or weak single particle vibronic excitons are also expected to be spectrally observable along with the collective state. The discussion of Sec. IV of paper I demonstrates the modulation sensitivity of such single particle resonances which are characterized by energies and oscillator strengths that depend on the lattice interaction $\mathbf{T}(\mathbf{k})$.

Absent from the theoretical treatment is a consideration of two and multiparticle states. The latter are attributable to indirect transitions from the crystal ground state, i. e., a particular multiparticle resonance results from the decay of a dipole-active single particle state. Every manifold of single particle vibronic excitons is accompanied by and coupled to manifolds of multiparticle states. An extended chain of interactions between the $O(0)$ $\rightarrow n(n)$ vibronic exciton and its corresponding multiparticle states is expected to profoundly affect the intensity distributions and energies of vibronic structure in strong coupling systems. Model calculations ${ }^{7}$ indicate that single particle vibronic polariton bandwidths shrink as a result of intensity stealing by multiparticle states. Equally important, single particle resonances broaden due to decay into multiparticle states and the observed spectra become diffuse and congested. Although there are no exciton calculations for systems like TCNQ and BDP which explicitly incorporate multiparticle resonances, the latter may be implicitly included in the single particle crystal dielectric function [Eq. (1.1)] by the introduction of a frequency-dependent damping term. This leads to modest success in calculating the spectra of TCNQ and BDP. ${ }^{2,6,8}$ The relevance of the foregoing to piezomodulation studies on strong coupling systems lies in the fact that polariton fission processes responsible for damping are sensitive to lattice strain. Hence, one may anticipate the appearance of features in modulated piezoreflection spectra which may be associated with multiparticle states. Strain is expected to modulate a vibronic exciton and its accompanying cloud of phonons.

TCNQ and BDP have been chosen for piezomodulated spectroscopic investigation because they are well-characterized strongly coupled systems. BDP has been intensively studied both experimentally and theoretically by Anex and Simpson, ${ }^{2}$ Fanconi, Gerhold, and Simpson, ${ }^{9}$ and Johnson and Simpson. ${ }^{6}$ Similarly, TCNQ has been studied experimentally by Pennelly and Eckhardt, ${ }^{1}$ and, recently, Philpott et al. ${ }^{10}$ have measured the $13 \mathrm{~K}$ reflection spectrum of the (010) face in an attempt to detect weak structure attributable to multiparticle excitations. These workers met with some success in resolving resonances which occur in the vicinity of the $O(0)$ and 0 (1) single particle states.

In addition to the amount of crystal spectroscopy data on TCNQ and BDP, there are additional reasons for pursuing an exploratory piezospectroscopic investigation of these systems. Crystals suitable for strain modulation may be readily obtained. The lowest energy singlet polariton spectra of crystalline TCNQ and BDP occur in the visible region of the spectrum which is experimentally easily accessible. Furthermore, the band systems of interest are reasonably well isolated from higher energy singlets. The latter is also advantageous from a theoretical standpoint in that one may neglect singlet-singlet configuration mixing. In this regard, it has been shown that the integrated intensities for the ${ }^{1} B_{3 u}-{ }^{1} A_{1 s}$ transition of TCNQ obtained from the most intense principal directions of the (001), (010), and (110) faces agree, within experimental error, with that obtained from the solution spectrum. ${ }^{11}$ Hence, one may initiate an analysis of the TCNQ piezomodulation spectra assuming that the entire ${ }^{1} B_{3 u}-{ }^{1} A_{1 g}$ electronic transition moment strength is partitioned into superimposed manifolds of single and multiparticle vibronic exciton transitions.

There are, however, several drawbacks associated with the study of TCNQ and BDP. Elastic constant data for these systems have not been obtained. Furthermore, no high pressure optical spectroscopy data exist for these systems. Hence, a discussion of piezomodulation results analogous to that presented for anthracene in paper III of this series is impossible. Lastly, theoretical calculations based on microscopic theory of the unmodulated reflection spectra of TCNQ and BDP by Pennelly and Eckhardt, ${ }^{1}$ Johnson and Simpson, ${ }^{6}$ and 
Philpott ${ }^{2}$ only approximately reproduce the observed structure. This is undoubtedly attributable to the complexity of vibronic interactions in these systems. Thus, one is forced to approach the analysis of the TCNQ and BDP piezomodulation results in a manner which differs from that developed previously.

We report in this paper the low resolution $\left(60 \mathrm{~cm}^{-1}\right.$ bandpass), polarized single crystal modulated piezoreflection spectra and the corresponding differential Kramers-Kronig transformed quantities obtained for the $a$ and $b$ principal directions of the (001) face of TCNQ and for the maximum reflecting principal direction (max.) of TCNQ (010) at $298 \mathrm{~K}$. Supplementing the latter are preliminary low resolution $\Delta R / R$ spectra obtained from the principal directions of BDP for (010). The polariton deformation potentials for these systems are also determined.

The paper is organized as follows: Experimental details are given in Sec. II. The latter also presents experimental results and the differential Kramers-Kronig transformed spectral profiles. Concluding discussion in Sec. III treats polariton strain sensitivity and features comparisons between measured and calculated results. In Sec. IV the role of piezomodulation spectroscopy in the study of molecular crystals is assessed.

\section{EXPERIMENTAL}

\section{A. Crystal preparation and physical properties}

Neutral TCNQ obtained from Eastman Organic Chemicals was purified by successive vacuum sublimations. The resulting pale yellow powder was used to prepare saturated solutions of TCNQ in either anhydrous acetone or chloroform. Slow evaporation of a crystal growth solution yielded well-formed crystals with a tabular habit. Thin platelets [plate face (001)] of TCNQ could be readily obtained and provided suitable specimens for modulated piezoreflection studies. Somewhat thicker crystals of TCNQ harvested from the growth solution after several weeks of slow evaporation could be used to secure thin platelets of the (010) face by cleaving. The TCNQ crystal morphology, face assignments, and principal directions have been reported. ${ }^{1}$ The max. principal direction for TCNQ (010) is entirely determined by the intense, long axis polarized ${ }^{1} B_{3 u}-{ }^{1} A_{18}$ molecular transition. ${ }^{1}$

Crystals of BDP suitable for piezospectroscopic examination were provided by M. R. Philpott. Crystal morphology, face assignments, and principal directions were found to coincide with those described by Neely. ${ }^{12}$

The general selection and mounting procedures utilized for TCNQ and BDP crystals were similar to those employed in the piezomodulation studies of PMDA-A and anthracene.

Crystals of TCNQ are monoclinic with a space group symmetry of $C 2 / c .{ }^{13}$ Projections of the molecules onto (001) and (010) are shown in Fig. 1. The four TCNQ molecules in the unit cell occur in translationally equivalent pairs.

Crystals of BDP have been reported to be monoclinic

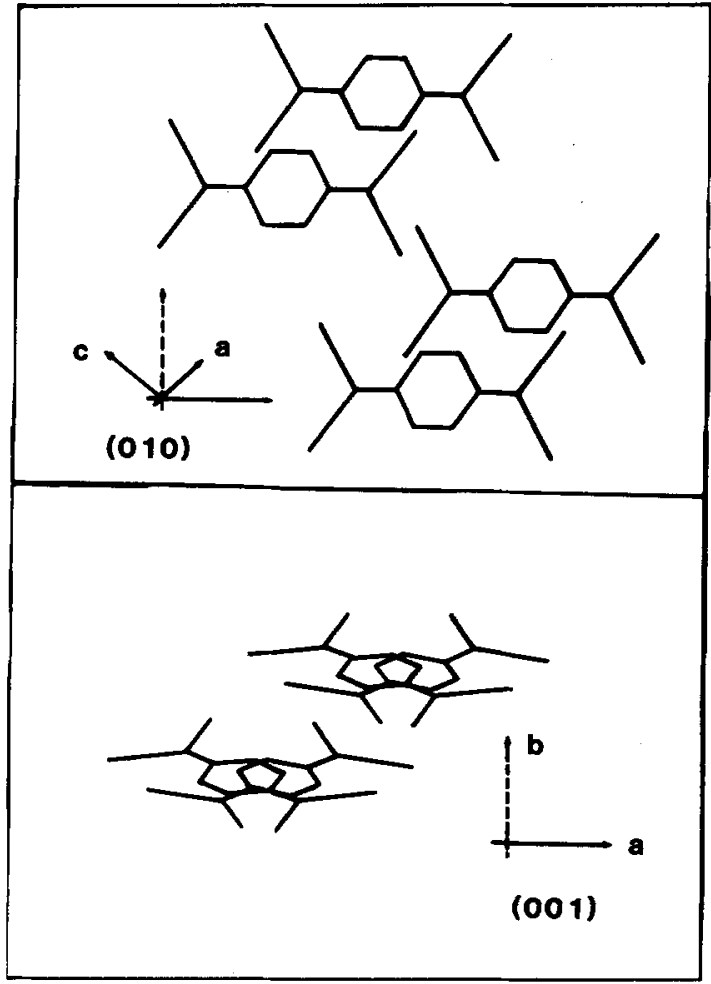

FIG. 1. Projection of TCNQ molecules onto (001) and (010).

by Neely ${ }^{12}$ and Selzer and Matthews. ${ }^{24}$ The space group symmetry is $C c$, and four translationally equivalent polymethinium ions with associated perchlorate counterions comprise the contents of the unit cell. The intense BDP transition of interest is polarized parallel to the polymethine chain. ${ }^{12}$

\section{B. Molecular spectroscopy information and site shifts}

The vapor phase absorption spectrum of TCNQ obtained at $\sim 463 \mathrm{~K}$ appears to be a single vibronic progression comprised of three prominent components. ${ }^{1}$ The $(0,0),(0,1)$, and $(0,2)$ vibronic transitions occur at 27620,28970 , and $30320 \mathrm{~cm}^{-1}$, respectively, and have corresponding Franck-Condon factors of 0.656 , 0.249 , and 0.099 . The progression spacing of $1350 \mathrm{~cm}$ $\mathrm{cm}^{-1}$ correlates well with reported Raman spectra. ${ }^{15,16}$ The solution absorption spectrum of TCNQ in chlorofor $\mathrm{m}^{1}$ at $298 \mathrm{~K}$ is shown in Fig. 2. The $(0,0)$ component of the vibronic progression occurs at $24880 \mathrm{~cm}^{-1}$ and a spacing of $1400 \mathrm{~cm}^{-1}$ is observed. The $(0,0)$, $(0,1)$, and $(0,2)$ vibronic transitions have Franck-Condon factors of $0.65,0.25$, and 0.10 , respectively, and the electronic transition dipole strength $d^{2}$ is reported to be 3. $36 \AA^{2}$ with a corresponding oscillator strength of 3. 06. The TCNQ absorption spectrum in a chlorobutane glass at $77 \mathrm{~K}^{1}$ is reasonably well resolved into five vibronic transitions which occur at $23900,24700,26100$, 27700 , and $28500 \mathrm{~cm}^{-1}$.

These single molecule spectral results may be used to estimate the van der Waals or site shift $D$ of the $\hat{\mathrm{L}}_{T}$ polarized TCNQ transition. The vapor-to-solution shift of the transition energy is $-2740 \mathrm{~cm}^{-1}$ whereas the vapor to-glass shift is $-3720 \mathrm{~cm}^{-1}$. This sense of the shift is 


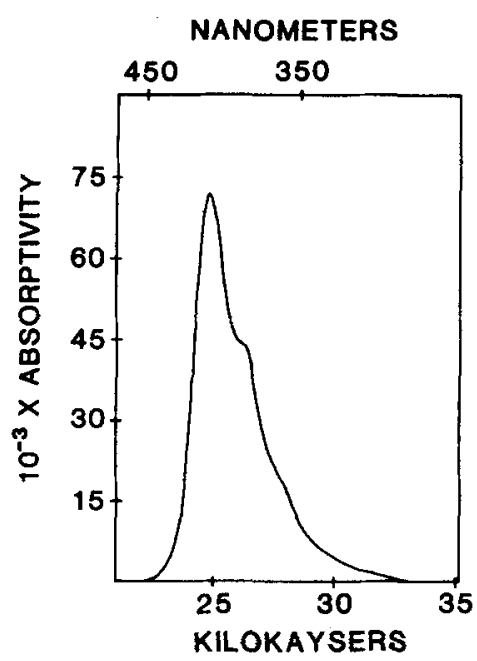

FIG. 2. The solution absorption spectrum of TCNQ in chloroform at $298 \mathrm{~K}$.

commonly observed for nonpolar organic molecules. The magnitudes are surprisingly large and exceed that reported in paper III for anthracene $\left(-1980 \mathrm{~cm}^{-1}\right)$. The strain modulation of the first singlet polariton in crystalline TCNQ may be expected to be dominated, as it is for anthracene, by the differential change in $D$. As will be seen, the data do not support this conjecture.

The single molecule absorption spectra of BDP have been reported by Anex and Simpson ${ }^{2}$ and Neely. ${ }^{12}$ The solution spectrum appears to have a single vibronic progression with a spacing of $\sim 1300 \mathrm{~cm}^{-1}$. The $(0,0)$ transition occurs at $24400 \mathrm{~cm}^{-1}$ and the electronic transition dipole strength $d^{2}$ is reported as $4.16 \AA^{2}$. The latter corresponds to a dipole length of $2.04 \AA$ and an os cillator strength of 3.31. Since the vapor phase spectrum of BDP is not obtainable, the site shift of the transition is not known with any degree of certainty. How-

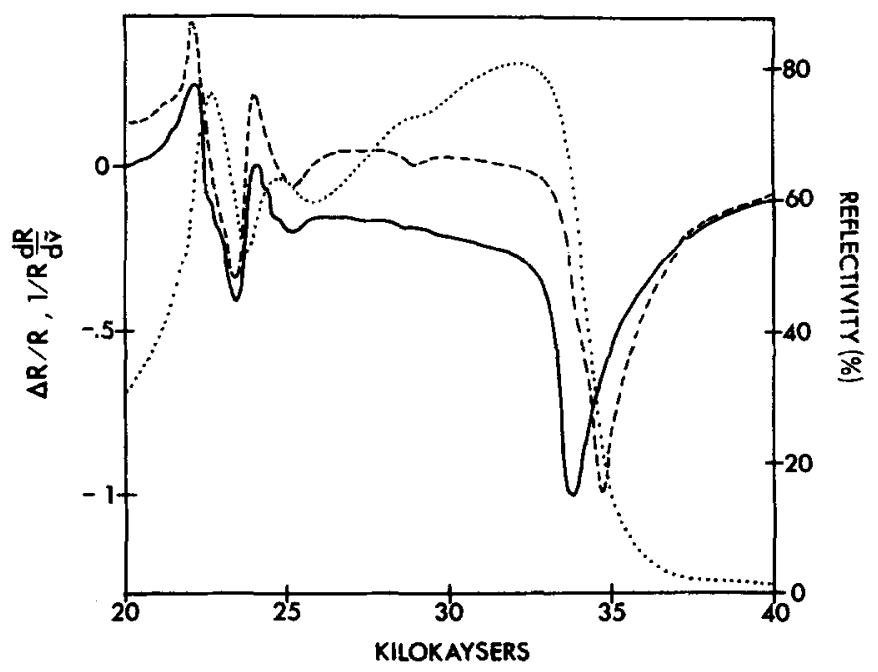

FIG. 3. Unmodulated reflection $R$ (dotted line), piezomodulated reflection spectrum $\Delta R / R$ (solid line), and calculated logarithmic derivative of the unmodulated reflection spectrum $(1 / R)(d R / d \tilde{V})$ (dashed line) for the maximally reflecting principal direction on $(010)$ of TCNQ. $\Delta R / R$ and $(1 / R)(d R / d \tilde{\nu})$ are shown normalized to facilitate comparisons.

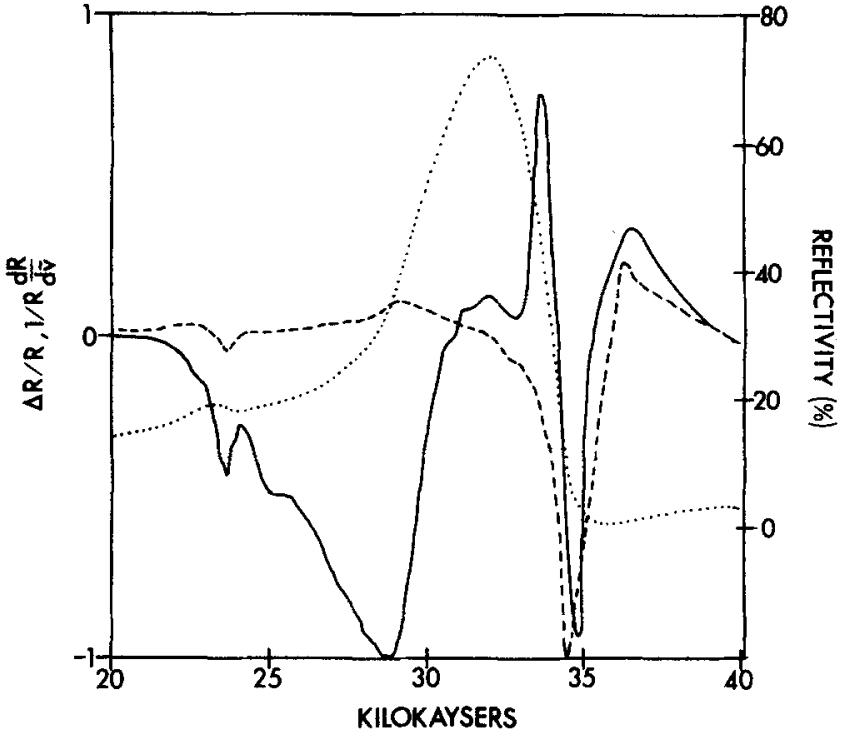

FIG. 4. $R, \Delta R / R$, and $(1 / R)(d R / d \tilde{D})$ spectra for the a polarization on (001) of TCNQ. (Labeling and plot conventions are the same as Fig. 3.)

ever, Philpott ${ }^{2}$ estimates a $D$ value for BDP in the range -1000 to $-1500 \mathrm{~cm}^{-1}$.

\section{Piezospectroscopic data}

\section{Piezoreflection spectra}

The low resolution superimposed $R, \Delta R / R$, and $(1 / R)(d R / d \bar{\nu})$ spectral profiles corresponding to the maximally reflecting (max.) principal direction of TCNQ on (010) are shown in Fig. 3. The same sequence of plotted spectral quantities is given in Fig. 4 for the a principal direction of TCNQ (001) and in Fig. 5 for the $b$ principal direction of TCNQ (001). Note that $\Delta R / R$ and $(1 / R)(d R / d \tilde{\nu})$ are shown normalized in Figs.

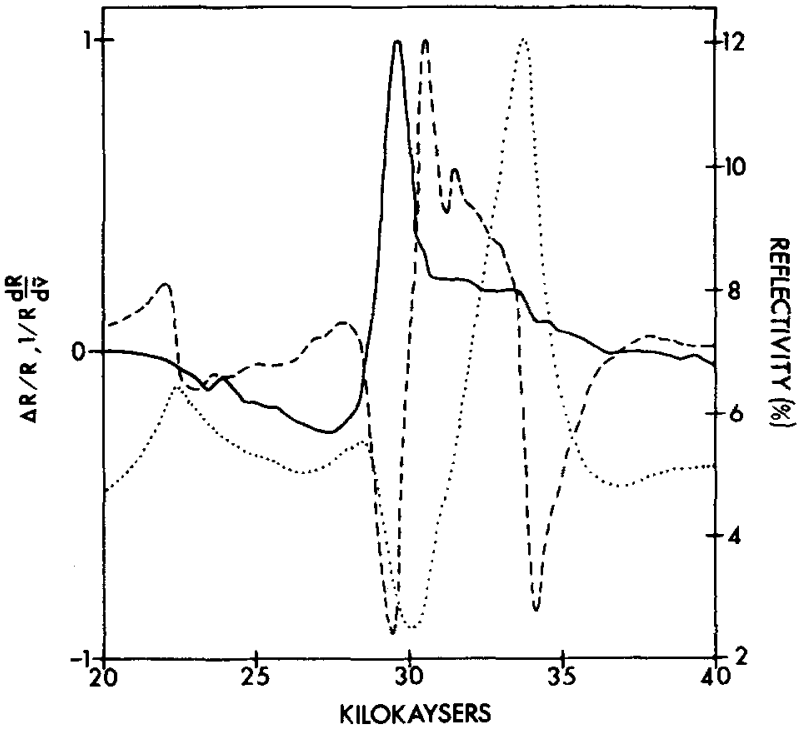

FYG. 5. $R, \Delta R / R$, and $(1 / R)(d R / d \tilde{\nu})$ spectra for the b polarization on (001) of TCNQ. (Labeling and plot conventions are the same as Fig. 3.) 


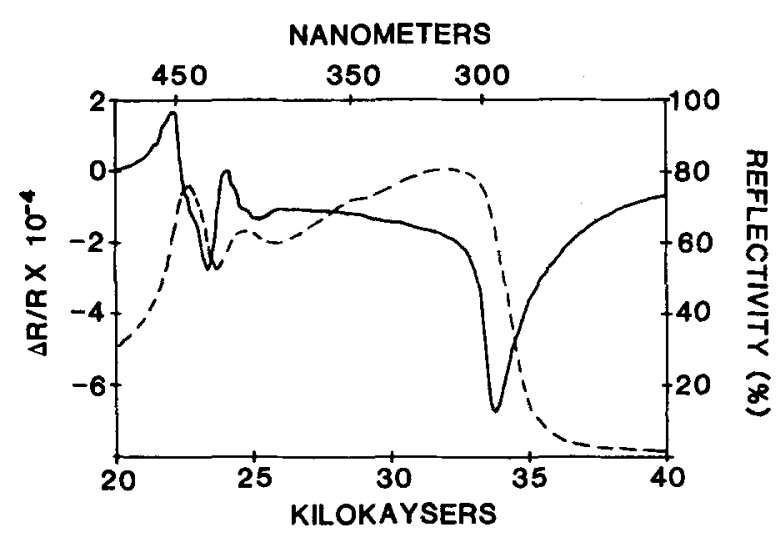

FIG. 6. $R$ and $\Delta R / R$ for the max. polarization on $(010)$ of TCNQ. Unmodulated spectrum (dashed line) and modulated spectrum (solid line).

3-5 to facilitate comparisons. Figure 6 displays the unnormalized $\Delta R / R$ spectrum for TCNQ (010) whereas Fig. 7 presents the unnormalized $a$ and $b$ polarized $\Delta R / R$ spectra obtained from the (001) face of TCNQ.

Examination of the $\Delta R / R$ and $(1 / R)(d R / d \bar{\nu})$ spectral profiles for the max. principal direction of TCNQ (010) indicates that the piezoreflection spectrum is primarily a logarithmic derivative with respect to the transition energy. These results for TCNQ (010) are similar to those obtained for PMDA-A and anthracene. However, in contrast to the latter, deviations between $\Delta R / R$ and $(1 / R)(d R / d \tilde{\nu})$ for TCNQ (010) are perceptible in the 32000 to $35000 \mathrm{~cm}^{-1}$ energy range. Although the precise origin of these deviations may only be determined by rigorous calculations and additional experiments, they imply, on the basis of theoretical considerations (paper I), that lattice strain does not result in a simple rigid shift of the entire stopping band. Both polariton bandwidth modulation together with strain-induced changes in individual vibronic polariton oscillator strengths can result in band shape distortions which yield the observed deviations. Since the deep minimum in $\Delta R / R$ at $33770 \mathrm{~cm}^{-1}$ occurs nearly at the inflection point observed in the $(1 / R)(d R / d \tilde{\nu})$ spectral profile, $\Delta R / R$ appears to be, in some respects, similar to the second derivative of the unmodulated reflection spectrum in this energy region.

In the energy region from 22100 to $29000 \mathrm{~cm}^{-1}$, the correspondence between $\Delta R / R$ and $(1 / R)(d R / d \bar{\nu})$ is quite good. Furthermore, both the sense (sign) of $\Delta R / R$ with respect to $(1 / R)(d R / d \vec{\nu})$ and comparable relative magnitudes of the excursions occurring in the two spectral profiles indicate that the single particle vibronic polaritons undergo approximately equal strain shifts to lower energy due to lattice compression. These strain shifts calculated from $\Delta R / R$ and $(1 / R)(d R / d \tilde{\nu})$ (see paper I) are reported in Table $I$.

The max. principal direction TCNQ (010) face spectrum shows weak structure superimposed on the major derivative-like excursions in $\Delta R / R$ from 22000 to 29000 $\mathrm{cm}^{-1}$. The energy spacings between successive spectral features of this type (Table II) agree reasonably well with several Raman active TCNQ molecular vibrational
TABLE I. Strain shifts of the lowest singlet exciton states in crystalline TCNQ and BDP from piezomodulation spectroscopy."

\begin{tabular}{|c|c|c|c|c|c|}
\hline \multirow[b]{2}{*}{ Crystal } & \multirow[b]{2}{*}{ Face } & \multirow{2}{*}{$\begin{array}{l}\text { Principal } \\
\text { direction }\end{array}$} & \multirow[b]{2}{*}{ Transition $^{b}$} & \multicolumn{2}{|c|}{$\begin{array}{l}\text { Strain shift } \\
\quad\left(\mathrm{cm}^{-1}\right)\end{array}$} \\
\hline & & & & $d$ & e \\
\hline TCNQ & $(010)$ & $\| \max$. & $\begin{array}{c}(0,0) \\
(0,1) \\
(0,2) \\
\text { Average }\end{array}$ & $\begin{array}{l}-0.48 \\
-0.41 \\
-0.38 \\
-0.42\end{array}$ & \\
\hline \multirow[t]{2}{*}{ TCNQ } & (001) & $\| \mathbf{a}$ & $\begin{array}{l}\text { Weak exciton; } \\
\tilde{\nu}=23.70 \mathrm{kK}\end{array}$ & -0.61 & -0.62 \\
\hline & & & $\begin{array}{l}\text { Collective exciton; } \\
\tilde{\nu}=30.20 \mathrm{kK}\end{array}$ & 1.01 & 1.08 \\
\hline \multirow[t]{3}{*}{ TCNQ } & (001) & $\| \mathrm{b}$ & $\begin{array}{l}\text { Weak exciton; } \\
\dot{D} \cong 23 \mathrm{kK}\end{array}$ & -0.54 & -0.45 \\
\hline & & & $\begin{array}{l}\text { Collective exciton; } \\
i=28.90 \mathrm{kK}\end{array}$ & & 1.83 \\
\hline & & & $\begin{array}{l}\text { Weak exciton; } \\
b=33.90 \mathrm{kK}\end{array}$ & -0.25 & -0.37 \\
\hline \multirow[t]{2}{*}{$\mathrm{BDP}$} & (010) & $\| \max$. & $(0,0)$ & -0.20 & \\
\hline & $(010)$ & $\| \min$. & $(0,0)$ & -0.18 & \\
\hline
\end{tabular}

The tabulated exciton-strain shifts correspond to a planar [either (001) or (010) face], isotropic, compressive crystal strain of $6.33 \times 10^{-5}$. Using the BDP lattice parameters of $a=12.15 \AA$ and $c=12.55 \AA$ from Neely (Ref. 12) the straininduced changes are $\Delta a=-7.69 \times 10^{-4} \AA$ and $\Delta c=-7.94 \times 10^{-4} \AA$.

${ }^{b}$ Refer to the text or appropriate table for additional information concerning the nature of the transition.

The estimated error in the strain shift is $\pm 0.1 \mathrm{~cm}^{-1}$.

The strain shifts in this column were calculated from the ratio of the change in $\Delta R / R$ measured from peak to trough in the vicinity of the transition to the corresponding change in $(1 / R)(d R / d \nu)$.

-The strain shifts in this column were calculated from the absorptivity and piezoabsorptivity data in a manner analogous to that described in footnote $d$.

energies. ${ }^{18}$ Hence, the $\Delta R / R$ spectrum appears to effectively resolve several weak two particle progressions. It is noteworthy that the interval values of the weak undulations in $\Delta R / R$ also agree quite well with corresponding intervals in structure reported by Phil-

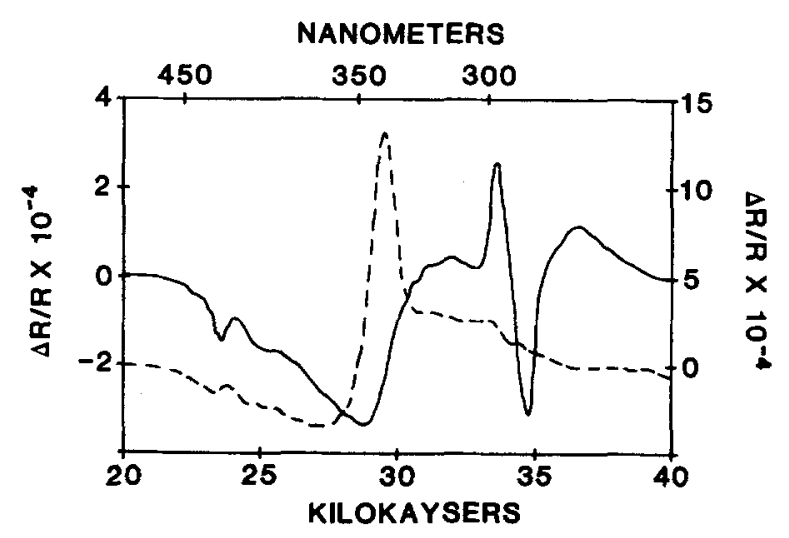

FIG. 7. $\Delta R / R$ spectra for the a (solid line) and b (dashed line) polarization on (001) of TCNQ. 
TABLE II. Frequencies of principal features of the TCNQ reflection and peizoreflection spectra for the max. principal direction of $(010),{ }^{a, b}$

\begin{tabular}{|c|c|c|c|}
\hline Description $^{c}$ & $\begin{array}{c}\text { Piezoreflection } \\
\tilde{\nu}(\mathrm{kK})\end{array}$ & $\begin{array}{l}\text { Reflection } \\
\tilde{\nu}(\mathrm{kK})\end{array}$ & Description ${ }^{c, d}$ \\
\hline $\max$. & 21.46 & & \\
\hline shl.; wk. shl. & $\begin{array}{c}21.70 ; 22.03 \\
22.12\end{array}$ & 21.65 & wk. shl. \\
\hline $\begin{array}{l}\text { br. } \max \text {. } \\
\text { wk. shl. }\end{array}$ & $\begin{array}{l}22.12 \\
22.47\end{array}$ & & \\
\hline shl. (infl.) & 22.62 & 22.60 & $\max .(76.2 \%)$ \\
\hline shl. & 22.86 & 23.10 & wk. shl. \\
\hline $\min$. & 23.41 & & \\
\hline infl. & 23.70 & 23.70 & $\min$. \\
\hline $\max$ & 24.04 & & \\
\hline shl. & 24.27 & & \\
\hline shl. & 24.69 & 24.70 & $\max .(63.4 \%)$ \\
\hline $\min$. & 25.22 & & \\
\hline shl. & 25.48 & 25.70 & $\min$. \\
\hline $\max$. & 25.94 & & \\
\hline infl. & 28.40 & 28.60 & $\max .(72.1 \%)$ \\
\hline wk. infl. & 30.50 & & \\
\hline infl. & 33.28 & 32.10 & $\max .(80.7 \%)$ \\
\hline $\min$. & 33.77 & 34.30 & infl. \\
\hline
\end{tabular}

${ }^{2}$ The crystal reflection spectrum was taken from Pennelly and Eckhardt (Ref, 1).

"The tabulated data spans the energy region corresponding to the vibronic progression of the long axis polarized, ${ }^{1} B_{3 u}-{ }^{1} A_{k}$ transition.

'Structure is designated as $\max .=$ maximum, $\min .=$ minimum, shl. $=$ shoulder, infl. $=$ inflection, wk. = weak, and br. = broad. ${ }^{d}$ Percentages given in parentheses represent absolute reflectivities at $\tilde{\nu}_{\max }$.

pott et al. ${ }^{10}$ from the $13 \mathrm{~K}$ unmodulated TCNQ (010) face reflection spectrum.

The piezoreflection results relevant to the $a$ and $b$ principal directions of TCNQ (001) shown in Figs. 4, 5 , and 7 represent the most striking effect of piezomodulation spectroscopy yet observed. Significant deviations between corresponding $\Delta R / R$ and $(1 / R)(d R / d \tilde{\nu})$

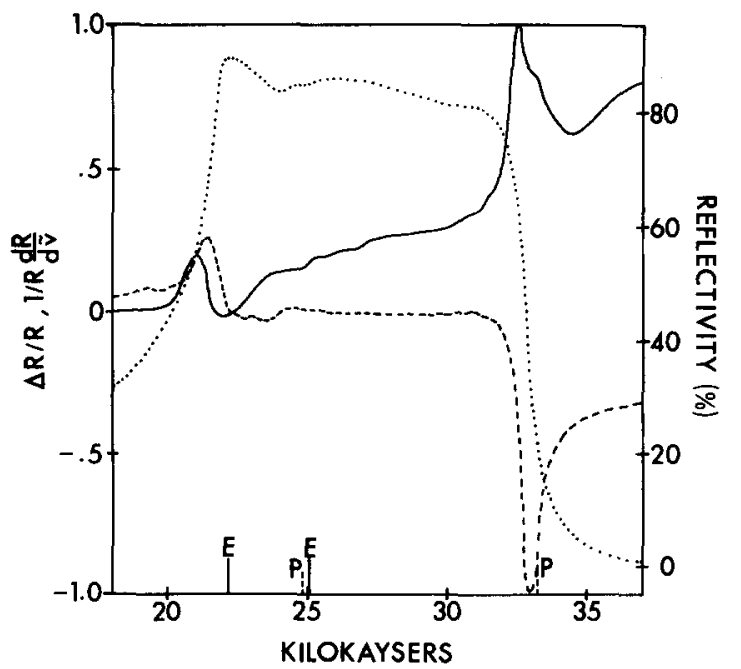

FIG. 8. $R, \Delta R / R$, and $(1 / R)(d R / d \tilde{\nu})$ spectra for the maximally (max.) reflecting principal direction on (010) of BDP. Calculated vibronic exciton $(E)$ and $\mathrm{k}=0$ polariton $(P)$ energies are shown (Ref. 2). (Labeling and plot conventions are the same as Fig. 3.)

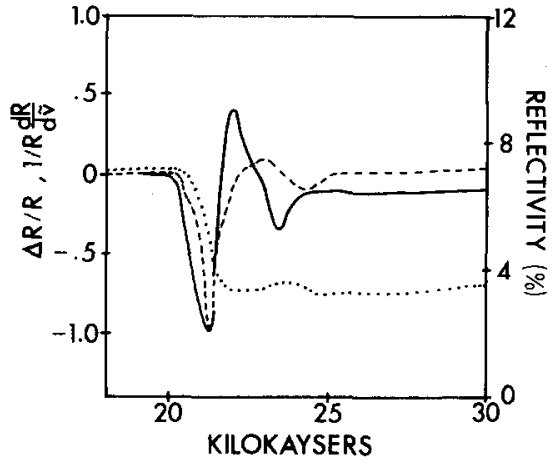

FIG. 9. $R, \Delta R / R$, and $(1 / R)(d R / d \tilde{\nu})$ spectra for the minimally (min.) reflecting principal direction on $(010)$ of BDP. (Labeling and plot conventions are the same as Fig. 3.)

spectral profiles are observed for both principal directions. In general, there are dramatic differences in both the magnitudes and signs of corresponding spectral features appearing in $\Delta R / R$ and $(1 / R)(d R / d \tilde{\nu})$. The unmodulated reflection (and Kramers-Kronig transformed absorption) spectra for the $a$ and $b$ principal directions clearly show the influence of a positive shifting intermolecular resonance interaction. Both factor group spectra reveal the existence of very strong vibronic coupling.

The low resolution superimposed $R, \Delta R / R$, and $(1 / R)(d R / d \tilde{\nu})$ spectral profiles corresponding to the max. and min. (minimally reflecting) principal directions of the (010) face of BDP at $298 \mathrm{~K}$ are shown in Figs. 8 and 9, respectively. The unmodulated reflection spectra were taken from Neely ${ }^{12}$ and the min. (010) spectra span the limited energy region in which structure is observed to occur in both the reflection and piezoreflection spectra. The very close similarity of the quasimetallic reflection spectra observed for the max. principal direction of TCNQ (010) and BDP (010) is clear and is typical of such crystals when $(\hat{\mu} \cdot \mathbf{k})=0$. The wide stopping bands observed in both systems arise from the excitation of transverse polaritons. The preliminary piezoreflection spectra were obtained for the max. and min. principal directions of BDP (010) in an attempt to ascertain whether the $\Delta R / R$ spectra of TCNQ and BDP agree as closely as the corresponding reflection data. It is evident from the normalized $\Delta R / R$ and $(1 / R)(d R / d \bar{\nu})$ spectral profiles shown for BDP (010) in Figs. 8 and 9 that polariton-strain coupling in BDP differs substantially from that observed in TCNQ (cf. Fig. 3).

The normalization constant of $4.83 \times 10^{-4}$ relevant to the BDP (010) $\max . \Delta R / R$ spectrum indicates that the excursions in $\Delta R / R$ for BDP are comparable to those observed in the TCNQ piezoreflection spectrum. Furthermore, both principal axis $\Delta R / R$ spectra for BDP $(010)$ exhibit a reasonably close cor respondence to the relevant logarithmic transition energy derivatives of $R$ in the 18000 to $23000 \mathrm{~cm}^{-1}$ region. At photon energies greater than $23000 \mathrm{~cm}^{-1}$, however, the max. $\Delta R / R$ spectrum of BDP (010) exhibits a line shape which is almost exactly opposite to that anticipated for a strict transition energy derivative. Similarly, the min. (010) 


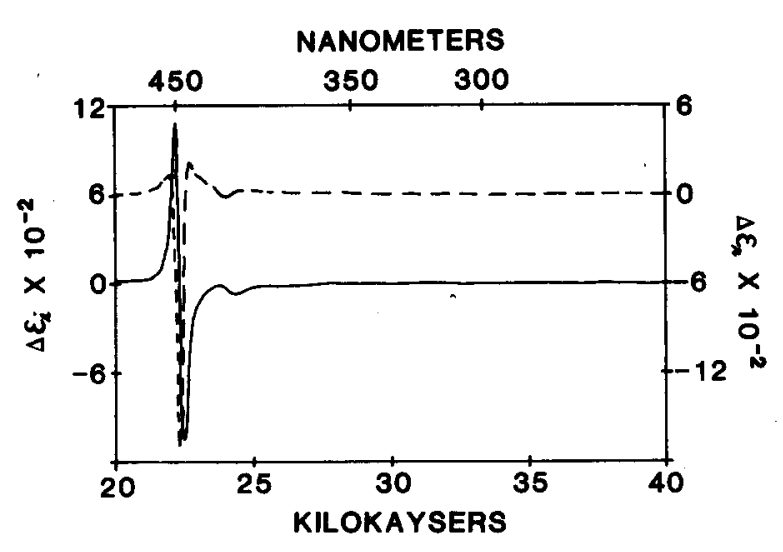

FIG. 10. $\Delta \epsilon_{r}$ (differential real part of dielectric function; dashed line) and $\Delta \epsilon_{i}$ (differential imaginary part of dielectric function; solid line) for the max. principal direction of (010) of TCNQ.

$\Delta R / R$ spectrum for BDP displays observable deviations from $(1 / R)(d R / d \tilde{\nu})$ in the 24000 to $26000 \mathrm{~cm}^{-1}$ region.

The vibronic structure present in the max. (010) $\Delta R / R$ spectrum of BDP is not as prominent as that observed for TCNQ (010). This is attributable to stronger vibronic coupling in BDP where in the collective exciton-polariton at $\approx 22000 \mathrm{~cm}^{-1}$ is characterized by an effective Franck-Condon factor of $\approx 0.95 .^{2}$ This collective level is split away from and lies below the threshold for multiparticle excitations. ${ }^{2,6.7}$ The latter appear in the max. (010) $\Delta R / R$ spectrum as either weak undulations or abrupt singular structure in the 23000 to $35000 \mathrm{~cm}^{-1}$ region. At $33300 \mathrm{~cm}^{-1}$, for example, the prominent shoulder in $\Delta R / R$ correlates well with similar structure obtained by difficult direct absorption measurements in the vicinity of the highest energy $k=0$ polariton branch. ${ }^{9}$

\section{Differential Kramers-Kronig spectra}

The differential dielectric function and absorbance spectra for the max. principal direction of TCNQ (010) are shown in Figs. 10 and 11 , respectively. The same sequence of data is repeated in Figs. 12 and 13 for the a principal direction of TCNQ (001) and in Figs. 14 and 15 for the $b$ principal direction of TCNQ (001). The

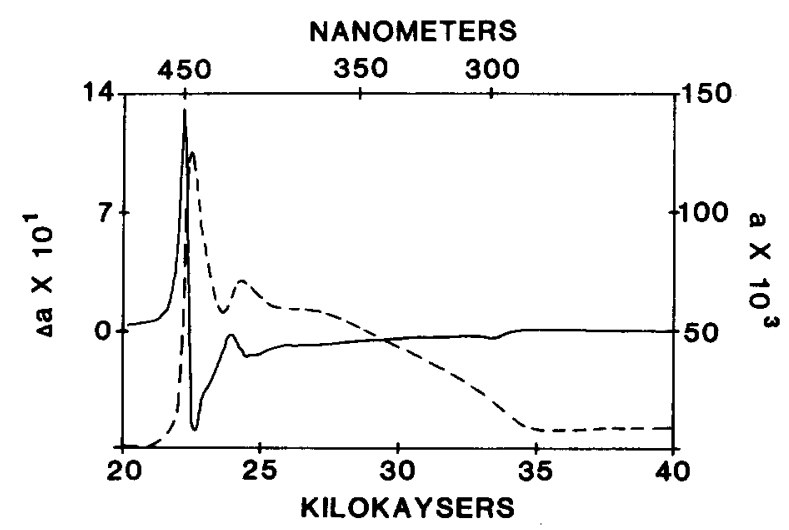

FIG. 11. a (dashed line) and $\Delta a$ (solid line) spectra for the max. principal direction of (010) of TCNQ.

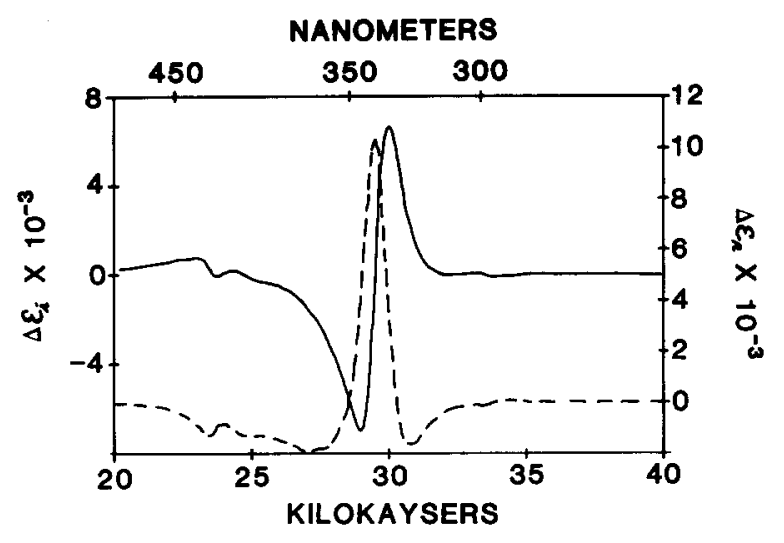

FIG. 12. $\Delta \epsilon_{r}$ and $\Delta \epsilon_{i}$ spectra for the a principal direction of (001) for TCNQ (convention as in Fig. 10).

transforms were obtained using the equations developed in paper I and the calculative procedure described in paper III.

In the low energy region (23000 to $28000 \mathrm{~cm}^{-1}$ ) of the a polarized $\Delta a$ spectrum, weak derivative-like structure characterized by a $1400 \mathrm{~cm}^{-1}$ progression is observed. As in the $\Delta R / R$ spectrum, these may be associated with weak single particle vibronic excitons which are broadened due to decay into a multiparticle continuum. In the 30000 to $35000 \mathrm{~cm}^{-1}$ region, a similar progression of weak structure is seen which is followed by a relatively prominent derivative-like excursion at $33800 \mathrm{~cm}$ $\mathrm{cm}^{-1}$. The $b$ polarized spectrum closely resembles the a polarized spectrum over the entire band. Thus, the progressions and shifts observed for the a polarization are seen in the $b$ polarization.

\section{DISCUSSION OF RESULTS}

According to semiclassical dipole theory, the polariton refers to a crystal resonance whose bandwidth is determined by both instantaneous (Coulomb) and retarded interactions. In the strong coupling regime where these effects are important, the solid state properties become dominant and obscure the molecular parentage of the resonances. In TCNQ and BDP these effects are most pronounced.

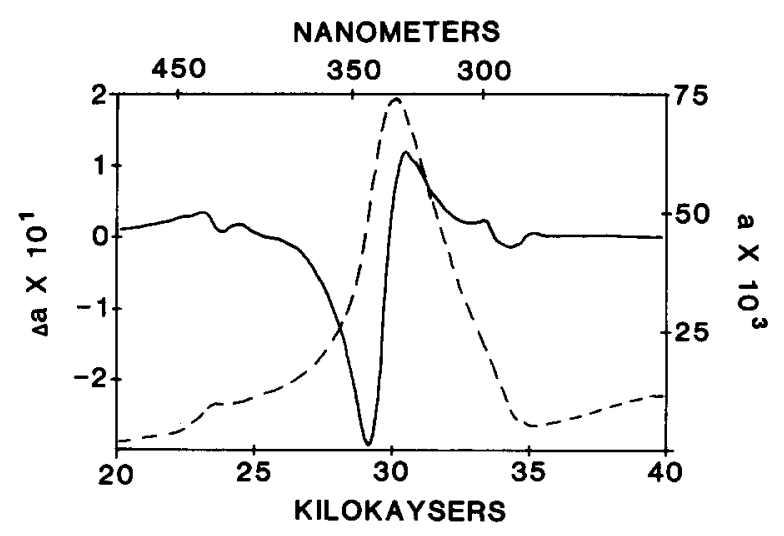

FIG. 13. $a$ (dashed line) and $\Delta a$ (solid line) spectra for the a principal direction of (001) for TCNQ. 
Because of different lattices, the two systems do not have the same type of interactions. TCNQ has factor group states for the $b$ axis and ac plane. BDP, however has only one exciton branch due to the translational equivalence of the four polymethenium ions in the unit cell.

For the max. principal direction on the $(010)$ face of both crystals the unmodulated reflection spectra indicate the existence of very strong exciton-photon coupling. In both cases, since $\hat{\mu} \cdot \hat{\mathbf{k}}=0$, the polariton modes are purely transverse.

The $\Delta a$ spectrum obtained for the max. principal direction of TCNQ (010) is very nearly a derivative of the unmodulated absorption spectrum. This indicates that the strain modulation of transverse polaritons in the $a b-$ sence of strong vibronic coupling is similar to that observed for the first singlet exciton of anthracene (paper III). It should be noted that the derivative-like excursions in $\Delta a$ at 22400 and $33700 \mathrm{~cm}^{-1}$ are opposite in sense and, furthermore, approximately define the stopping bandwidth. This is taken as an indication of stop band modulation. Specifically, a lattice compression results in a red shift of the lowest energy $k \rightarrow \infty$ polariton and a simultaneous blue shift of the highest energy $\mathbf{k}=0$ polariton.

These observations are in accord with notions concerning polariton modulation given in paper I. As previously noted, the piezoreflection spectra observed for the max. principal directions of TCNQ and BDP (010) differ significantly. Examination of the theoretically determined piezoreflection spectra for the two level system given in Fig. 4 of paper I indicates that the two crystals have different modulation parameters dominating their piezomodulation responses. In the case of TCNQ, the measured $\Delta R / R$ agrees extremely well with the calculated piezomodulation spectrum where the response is the expected composite of variances in the exciton resonance frequency, the plasma frequency, background dielectric constant, and oscillator strength [cf. Fig. 4(a) of paper I]. In contrast, the dominating modulation parameter observed in the $\Delta R / R$ spectrum of $\mathrm{BDP}$ is the strain-induced oscillation of the plasma frequency [cf. Fig. 4(c) of paper I]. Hence, the modulation is primarily that of the stopping bandwidth. In

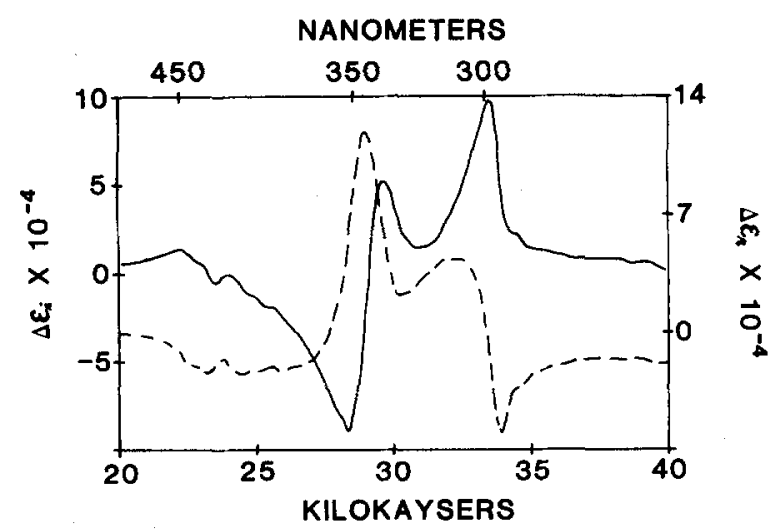

FIG. 14. $\Delta \epsilon_{\tau}$ and $\Delta \epsilon_{i}$ spectra for the $b$ principal direction of (001) for TCNQ (convention as in Fig. 10).

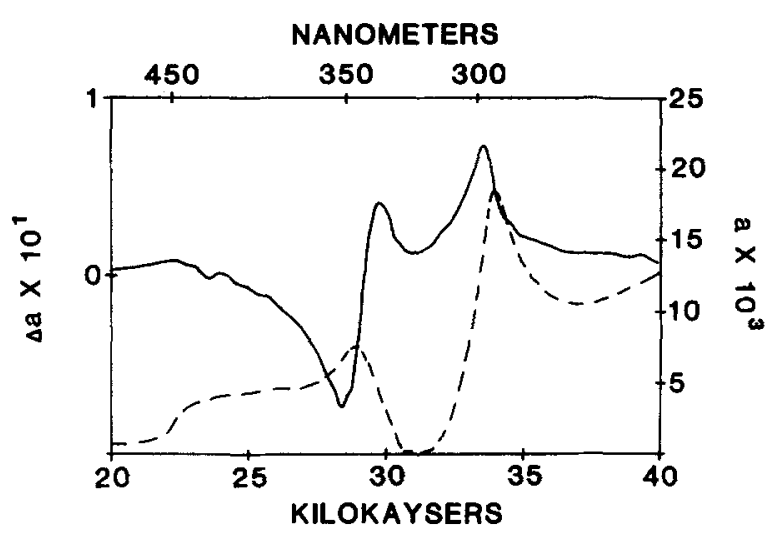

FIG. 15. $a$ (dashed line) and $\Delta a$ (solid line) spectra for the b principal direction of (001) for TCNQ.

both crystals, sharp structure appears in $\Delta R / R$ in the region of the lowest energy $\mathbf{k} \rightarrow \infty$ polariton. Furthermore, the correspondence between $\Delta R / R$ and

$(1 / R)(d R / d \bar{\nu})$ in this energy region is quite good. Conversely, such correspondence may deteriorate dramatically (as in BDP) in the vicinity of the highest energy $\mathbf{k}=0$ polariton where the $\Delta R / R$ spectrum may be comprised of two or more differentials which are comparable in magnitude but opposite in sign. Thus, the observed behavior of strongly coupled crystals with purely trans verse exciton-polaritons is reasonably described by semiclassical dipole theory. Furthermore, the latter clearly facilitates the recognition of relative contributions to $\Delta R / R$ from various exciton parameters appearing in the dielectric function. Further discussion will now focus on the piezomodulation data presented for TCNQ.

It is in the piezomodulation spectra of the (001) face for TCNQ where the most unusual features are found. Some deviations from a strict logarithmic energy derivative are expected because the vibronic polaritons observed in the a polarized TCNQ (001) face spectrum are mixed transverse-longitudinal excitations. ${ }^{17}$ It is reasonable that a lattice strain modulates the macroscopic longitudinal and transverse coupling energies differently. This is predicated on the idea that the macroscopic longitudinal and transverse dipole interactions are effectively modified or screened by different background constants $\epsilon_{0}^{r}$ and $\epsilon_{0}^{L}$, respectively. The single particle crystal dielectric function [Eq. (1.1)] is only appropriate to transverse polaritons. Hence, the inclusion of a scalar background dielectric constant $\epsilon_{0}$ is reasonable. ${ }^{4}$ However, since similar deviations between $\Delta R / R$ and $(1 / R)(d R / d \tilde{\nu})$ are observed in both the $a$ and $\mathrm{b}$ polarized (the b polarized polaritons are transverse) TCNQ (001) face spectra, one must conclude that they arise from ef fetcs in common to both principal directions. In this regard, the dipole wave sums $I(k)$ reported by both Pennelly ${ }^{11}$ and Johnson and Simpson ${ }^{6}$ are large and positive for both the $b$ and ac polarized factor group components of the first singlet system. The unmodulated reflection and absorption spectra for both the $a$ and $b$ principal directions unmistakably reflect the influence of a positive intermolecular resonance interaction. Both factor group spectra reveal the existence of very strong vi- 
bronic coupling. The recognizable collective vibronic exciton is significantly blue shifted relative to the corresponding collective resonance observed in the max. direction of TCNQ (010). Furthermore, the weak or hypochromic vibronic excitons predicted from the single particle theory ${ }^{4}$ lie to the red of the collective excitation in both factor group spectra.

There is strong evidence in the $R$ and $\Delta R / R$ a and $\mathrm{b}$ polarized spectra for the existence of multiparticle excitations. Insofar as the reflection spectra are concerned, the pronounced observable structure which may be described in terms of single particle vibronic polaritons is broad and diffuse and is superimposed on an apparent continuum of multiparticle excitations. The spectral features in the piezoreflection spectra facilitate the recognition of progressions in a considerable amount of weak structure characterized by energy spacings which correlate well with known Raman active TCNQ vibrations, Hence, heretofore unobservable multiparticle excitations are successfully detected in the piezomodulation spectra.

On the basis of preceding considerations, the unusual $\Delta R / R$ spectra observed for both the $a$ and $\mathrm{b}$ principal directions appear to be attributable to the strain modulation of the very strong vibronic coupling of molecular polaritons which is extant and easily observable for the unstrained crystal. Both the pronounced and weak excursions registered in the piezoreflection spectra appear to define threshold and singularities in the superimposed single and multiparticle exciton bands. This observation will have to be reinforced by higher resolu tion $\Delta R / R$ spectra taken at lower temperature. Confirmation will mean that deconvolution of badly overlapped single particle and multiparticle progressions will then be possible together with accurate determination of the differences in the correspond ing ground and excited state deformation potentials.

The apparent exciton strain shifts obtained from the TCNQ (001) and (010) spectra listed in Table I are, because of these additional effects, at best, estimates. The correspondence between $\Delta R / R$ and $(1 / R)(d R / d \tilde{\nu})$ is close enough to obtain fairly reliable strain shifts from the data only in the energy regions of the weak single particle resonances observed in the TCNQ (001) spectra. For TCNQ and BDP (010), advantage is taken of the close correspondence between $\Delta R / R$ and $(1 / R)(d R / d \tilde{\nu})$ in the vicinity of the lowest energy $k \rightarrow \infty$ polariton to secure reasonable strain shift values.

In some respects, the differential Kramers-Kronig transformed piezoabsorption spectra provide a picture of polariton-strain coupling in TCNQ which is somewhat easier to understand than that afforded by an inspection of the $\Delta R / R$ data. In the low energy region (23000 to $28000 \mathrm{~cm}^{-1}$ ) of the a polarized differential absorbance $(\Delta a)$ spectrum, weak derivative-like structure characterized by an approximately $1400 \mathrm{~cm}^{-1}$ energy spacing is observed. These features may be correlated with weak single particle vibronic excitions. The collective vibronic exciton is approximately located by the inflection in at $29900 \mathrm{~cm}^{-1}$. The energy spacing between the center of gravity of the manifold of the weak exciton transitions and the collective state is approximately $4600 \mathrm{~cm}^{-1}$. Within the context of the single particle vibronic coupling formalism (paper I and Ref. 4), the spacing of $4600 \mathrm{~cm}^{-1}$ is taken equal to the electronic transition dipole coupling energy $I(k)$ assuming an effective Franck-Condon factor of unity for the collective state. This measured value for $I(k)$ for the ac polarized factor group component agrees favorably with the calculated values of Johnson and Simpson. ${ }^{6}$

In the energy region from 30000 to $35000 \mathrm{~cm}^{-1}$ in the a polarized $\Delta a$ spectrum a progression of weak structure is observed followed by a relatively prominant derivative-like excursion at $33800 \mathrm{~cm}^{-1}$. The general trend observed in this energy region is strikingly similar to that previously noted for the single particle vibronic excitons. Specifically, a progression of relatively weak resonances lies to the red of and is split from a moderately intense transition. Thus, the structure in the 30000 to $35000 \mathrm{~cm}^{-1}$ energy region is due to weak two particle excitations split from a collective two particle state. This assignment provides experimental verification of at least one feature predicted by Philpott's ${ }^{7}$ vibronic coupling theories.

The sense of the derivative-like excursions in both spectra indicates that the weak resonances associated with them undergo comparable red shifts due to lattice compression. In contrast, the sense of the derivativelike structure in the vicinity of the collective vibronic exciton for both principal directions indicates that this resonance apparently undergoes a blue shift due to lattice compression. This phenomenon has not been observed for any other band system or transition considered in piezomodulated systems studied so far. This sense inversion is quite surprising in view of the rather large, negative, site shift $D$ for the $\hat{\mathrm{L}}_{T}$ polarized TCNQ transition. The present data do, however, indicate rather strong coupling and hence decay of the single particle collective mode into multiparticle excitations.

The strain shift data (Table I) show that all of the measured strain shifts are negative with the exception of those relevant to the collective vibronic exciton observed in the $a$ and $b$ polarized spectra of TCNQ (001). It should be emphasized that these shifts are not as reliable as those reported for the PMDA-A and anthracene systems. This is particularly true for those shifts derived from the TCNQ (001) piezomodulation spectra. In general, the easily perceptible deviations of $\Delta R / R$ (or $\Delta a$ ) spectra from strict transition energy derivatives vitiate, to some extent, the efficacy of the simple calculative procedures used to determine the strain shifts. Thus, polariton-strain coupling apparently involves several concurrently operative mechanisms which result in band shape distortion and oscillator strength changes as well as simple energy shifts. Indeed, even within the context of the semiclassical dipole theory relevant to single particle vibronic polaritons, the crys tal dielectric function is given in terms of at least five potentially strain-sensitive parameters: $\epsilon_{0}, \gamma_{u}, \Omega_{u}^{2}, F_{u}$, and $\omega_{0}^{2}$ (see paper I). In some instances, the only real certainty may be the sign or direction of the strain shifts Measured and calculated strain shifts may be compared 
TABLE III. Calculated strain energies and shifts for the first singlet transition of crystalline TC NQ. ${ }^{2}$

\begin{tabular}{|c|c|c|c|c|c|c|c|}
\hline Face & $\begin{array}{l}\text { Principal } \\
\text { direction }\end{array}$ & $\begin{array}{l}I(\mathrm{kj})^{\mathrm{b}} \\
(\mathrm{kK})\end{array}$ & $\begin{array}{l}\text { Unstrained } \\
\text { energy }(k K)\end{array}$ & $\begin{array}{l}\text { Strained } \\
\text { energy }(k K)\end{array}$ & $\begin{array}{l}\text { Strain } \\
\text { shift } \\
\left(\mathrm{cm}^{-1}\right)\end{array}$ & $\begin{array}{l}\text { Mean strain } \\
\text { shift and } \Delta w^{\mathrm{d}} \\
\quad\left(\mathrm{cm}^{-1}\right)\end{array}$ & $\begin{array}{l}\text { Oscillator } \\
\text { strengths }\end{array}$ \\
\hline \multirow[t]{4}{*}{$(010)$} & \multirow[t]{4}{*}{$\| \max }$. & \multirow[t]{4}{*}{-1.8355} & $22.22354(E)^{t}$ & $22.22248(E)$ & $-1.06(E)$ & \multirow{4}{*}{$\begin{array}{r}-0.36 \\
(1.40)\end{array}$} & 2.9077044 \\
\hline & & & $25.04267(P)$ & $22.04192(P)$ & $-0.75(P)$ & & $\left(2.94 \times 10^{-5}\right)$ \\
\hline & & & $25.21990(E)$ & $25.21914(E)$ & $-0.76(E)$ & & 0.15329492 \\
\hline & & & $34.15097(P)$ & $34.15131(P)$ & $0.34(P)$ & & $\left(-2.94 \times 10^{-5}\right)$ \\
\hline \multirow[t]{4}{*}{$(001)$} & \multirow[t]{4}{*}{ If $\mathbf{a}$} & \multirow[t]{4}{*}{4.4541} & $24.99404(E)^{t}$ & $24.99292(E)$ & $-1.12(E)$ & & 0.05572516 \\
\hline & & & $25.04267(P)$ & $25.04154(P)$ & $-1.13(P)$ & -0.23 & $\left(-1.90 \times 10^{-5}\right)$ \\
\hline & & & $29.26814(E)$ & $29.26763(E)$ & $-0.51(E)$ & $(0.57)$ & 3.0052741 \\
\hline & & & $34.15097(P)$ & $34.15103(P)$ & $0.06(P)$ & & $\left(1.90 \times 10^{-5}\right)$ \\
\hline
\end{tabular}

${ }^{a}$ Only the $(0,0)$ and $(0,1)$ vibronic components of the ${ }^{1} B_{3 u}-{ }^{1} A_{1}$ transition were considered in these model calculations for TCNQ. The calculative procedures together with the relevant equations and definitions may be found in paper $\mathrm{I}$. The free molecule spectroscopic parameters used in the calculations are $\tilde{\nu}_{0,0}=27.62 \mathrm{kK}$ and $\nu_{0,1}=28.97 \mathrm{kK} ; \xi_{0,0}^{2} \cong 0.7$ and $\xi_{0,1}^{2} \cong 0.3 ; f_{0,0} \cong 2.11$ and $f_{0,1} \cong 0.951$. The Franck-Condon factors and oscillator strengths represent approximate values used to facilitate the two-level calculations. Eckhardt and Pennelly (Ref. 1) report Franck-Condon factors of $0.658,0.249$, and 0.999 for the $(0,0),(0,1)$, and $(0,2)$ transitions, respectively. These workers also report an electronic transition dipole strength of $3.36 \AA^{2}$.

${ }^{b}$ The $I(k)$ values are those relevant to the ac polarized vibronic exciton states. The analytic part of $I(k)$ was approximated by a modified, cubic crystal Lorentz factor of $-0.143[=t(\mathrm{k})]$. A background dielectric constant $\epsilon_{0}$ of 1.5 was used to "screen" the dipole interactions.

'The Coulomb exciton and $\mathrm{k}=0$ polariton energies are designated by $(E)$ and $(P)$, respectively.

The numbers in parentheses represent the strain-induced changes in the polariton (or Coulomb exciton) bandwidth $\Delta w$. Refer to the text for additional information concerning both $\Delta w$ and the mean polariton strain shifts.

${ }^{\theta}$ The strain-induced changes in the vibronic exciton oscillator strengths are given in parentheses. The total crystal oscillator strength $F_{0,0}+F_{0,1}$ is 3.061 .

'The measured exciton and $\mathrm{k}=0$ polariton energies for the unstrained crystal are in good agreement with these values.

for the strong coupling regime keeping these caveats in mind.

The strain-induced changes in the site shift of the $\hat{\mathbf{L}}_{T}$ polarized transition corresponding to a $D$ value of $-3480 \mathrm{~cm}^{-1}$ were used in conjunction with formulas (A1)-(A3) given in paper I, to perform model calcula tions for TCNQ using reported dispersion energy calculations. For simplicity, the TCNQ (001) and (010) spectra were assumed to be comprised of only two vibronic polariton transitions. Although this is admittedly simplistic, the measured spectra do indicate that the bulk of the crystal electronic transition intensity may be approximately partitioned into two or three single particle vibronic excitations. A summary of calculated exciton energies and oscillator strengths together with the corresponding strain-induced changes is given in Table III. The exciton (polariton) energies and corresponding oscillator strengths determined for the unstrained crystal provide a realistic description of the unmodulated crystal absorption spectra for the max. principal direction of the (010) face and the a principal direction of the (001) face.

For the max. principal direction of TCNQ (010), there is good agreement between theoretical and experimental findings. All of the calculated strain shifts are negative, i. e., red shifts with the exception of the highest energy $\mathbf{k}=0$ polariton. This implies that lattice compression produces an increase in the width of the stop band. The piezoabsorption data for this polarization confirm that the experimental findings are consistent with the calcu- lated results. Furthermore, the average measured exciton-strain shift for TCNQ (010) of $-0.42 \mathrm{~cm}^{-1}$ agrees satisfactorily with the calculated mean shift of -0.36 $\mathrm{cm}^{-1}$. The latter represents the strain shift of the center of gravity of the polariton band.

Agreement between calculated and measured shifts for the a principal direction of TCNQ (001) is not as good as that obtained for TCNQ (010) but this is not particularly surprising in view of the strong vibronic coupling effects evident in the TCNQ (001) face spectra. A further demonstration of this is shown in Fig. 16, where the calculation of the a polarized spectrum is shown. This curve, based on paper I, Sec. IV and Appendix, shows the separate effects due to modulation of $\Omega_{u}, \omega_{0}$, $\epsilon_{0}$, and $F_{u}$. Comparison to the experimental a polarized piezomodulation shows reasonable agreement only in the single particle region. The multiparticle region shows disagreement to the extent that even the sense of the derivatives are opposite. The failure of the two level model for the mixed transverse-longitudinal situation indicates that the role of multiparticle states must be as sessed further.

A similar calculation for the completely transverse (101) spectrum is shown in Fig. 4 of paper I. Here the agreement with the experimental modulated piezoreflection spectrum is quite good. This leads to the conclusion that the strain modulation of transverse vibronic polaritons results in a reasonably simple piezomodulation spectrum which fits the two level model treated in paper I. 


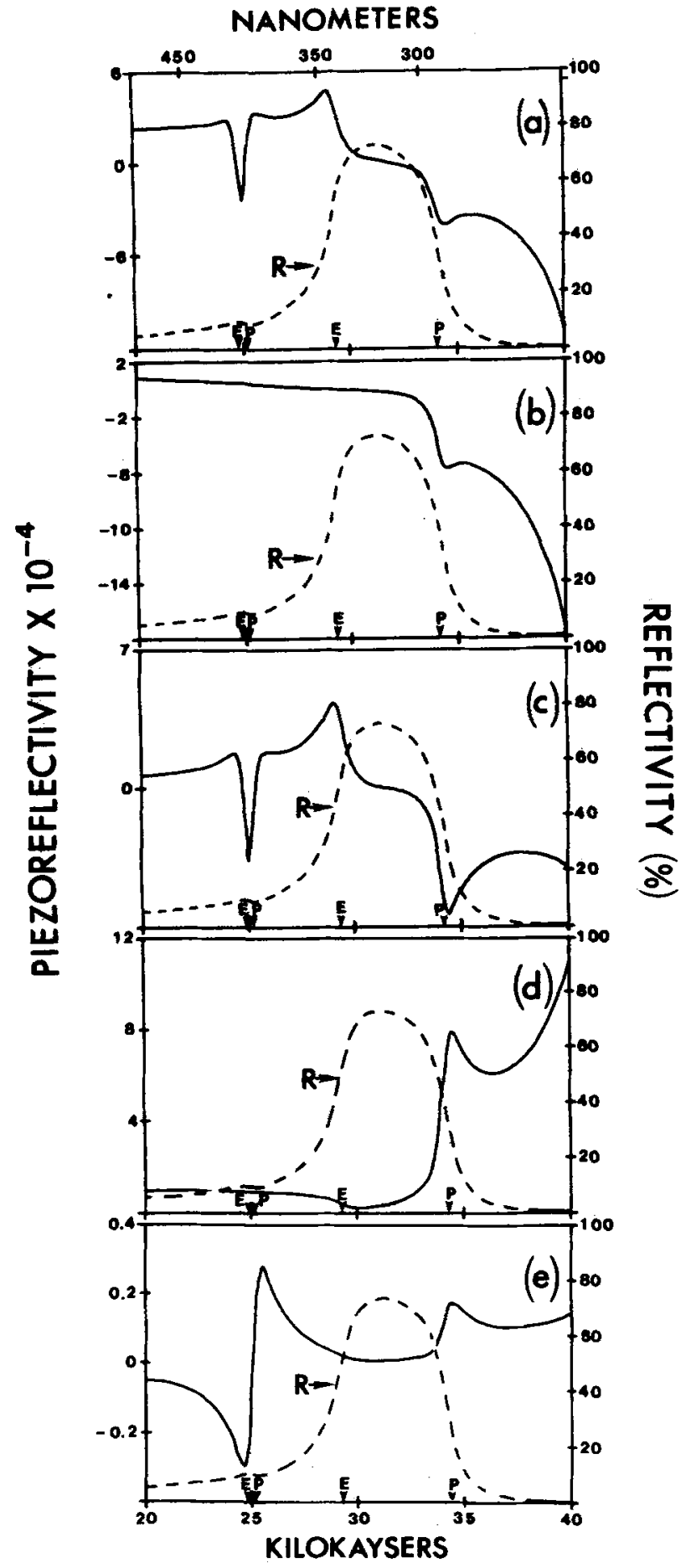

FIG. 16. Calculated piezoreflection spectra for the a polarization of TCNQ (001). Figure 16(a) shows the composite piezoreflection spectrum (solid line) corresponding to the model reflection band (dashed line). Figures $16(\mathrm{~b}), 16(\mathrm{c}), 16(\mathrm{~d})$, and $16(\mathrm{e})$ correspond to $(\Delta R / R)_{D}=(1 / R)(B R / \partial P) d P$, where the modulation parameter $P$ is $\epsilon_{0}, \Omega_{u}^{2}, \omega_{0}^{2}$, and $F_{u}$, respectively.

\section{CONCLUSIONS}

The exploratory piezospectroscopic investigation of the lowest energy singlet polariton states in TCNQ and BDP has yielded spectral information heretofore unattainable by conventional spectroscopic techniques at equivalent temperature. Most notably, the piezomodula- tion spectra are rich in structure which may be correlated with single and multiparticle excitations. This presents the possibility of resolving or effectively deconvoluting badly overlapped vibronic progressions in strong coupling systems.

In a broad context, piezomodulation spectroscopy has shown that it is a direct measure of intermolecular excitation resonance in molecular crystals. As the progression is made from an essentially oriented gas system (pyromellitic dianhydride-anthracene) to the intermediate coupling system (anthracene) to the strong coupling TCNQ and BDP systems, it is apparent that the measured piezoreflection spectrum deviates more and more from the calculated logarithmic derivative. From another point of view, as the exciton band structure becomes more important, the observable strain effects become more pronounced.

The extreme deviation of the measured piezomodulation spectrum from that of the calculated spectrum in the case of mixed transverse-longitudinal excitations indicates that the role of multiparticle states is considerable. Because of the change in sense of the piezomodulation spectrum from that of the calculated spectrum, the response of the multiparticle states may be quite different from that of the single particle states. Along this line, some attention must be given to the experimental results which indicate abnormally high sound absorption by molecular crystals. ${ }^{18,19}$ Theoretical treatment of the phenomenon shows that, allowing for anharmonicity, phonon processes of high order are likely. Of particular importance are three quantum processes between acoustic and optical phonons. ${ }^{19}$ Thus, additional studies on strongly coupled systems as a function of drive frequency and temperature are necessary.

The piezomodulation is an effect which affects most directly and strongly the solid state interactions in molecular crystals. As demonstrated in paper I, the molecular potentials are unaffected in the strain regime of the experiment. Thus, the piezomodulation may prove to be an effective perturbation not only for the spectroscopy of molecular crystals but for other solid state properties such as conductivity.

The investigations in this series of papers have provided an initial survey of the possibilities of piezomodulation spectroscopy of molecular crystals. The results have been interpreted on a simple physical basis. The results demonstrate that piezomodulation spectroscopy, especially in the strong coupling regime, is an area of great potential and will provide the basis for future investigations in the study of molecular crystals.

${ }^{1}$ R. R. Pennelly and C. J. Eckhardt, Chem. Phys. 12, 89 (1975).

${ }^{2}$ M. R. Philpott, J. Chem. Phys. 54, 2120 (1971); B. G. Anex and W. T. Simpson, Rev. Mod. Phys, 32, 466 (1960).

${ }^{3}$ W. T. Simpson and D. L. Peterson, J. Chem. Phys. 26, 588 (1957).

"M. R. Philpott, in Advances in Chemical Physics, edited by I. Prigogine and S. A. Rice (Academic, New York, 1973), Vol. 23 , p. 227 . 
${ }^{5}$ G. D. Mahan, J. Chem. Phya. 41, 2930 (1964); 48, 1569 (1965).

${ }^{6}$ B. B. Johnson and W. T. Simpison, J. Chem. Phys. 65, 4246 (1876).

7M. R. Philpott, J. Chem. Phys. 47, 4437 (1967); 55, 2039 (1971).

${ }^{8}$ M. R. Philpott, J. Chem. Phya. 62, 5842 (1970).

B. M. Fanconi, G. A. Gerhold, and W. T. Simpson, Mol. Cryst. Liq. Cryst. 6, 41 (1969).

${ }^{10}$ M. R. Philpott, P. M. Grant, K. Syassen, and J. M. Turlet, J. Chem. Phys. 67, 4229 (1977).

${ }^{11}$ R. R. Pennelly, Ph.D. thesis, University of Nebraska, 1975.

128. Neely, Ph. D. thesis, Yale University, 1965.

${ }^{13}$ R. E. Long, R. A. Sparks, and K. N. Trueblood, Acta
Cryataliogr. 18, 932 (1965).

14J. O. Selzer and B. W. Matthews, J. Phys. Chem. 80, 631 (1976).

${ }^{15} \mathrm{~T}$. Takenaks, Spectrochim. Aata Part A 27, 1753 (1971).

${ }^{16}$ A. Girlando and C. Pecile, Speotrochim. Acta Part A 29, 1859 (1973).

${ }^{17}$ V. M. Agranovioh and V. L. Ginaberg, Spatial Dispersion in Crystal Optics and the Theory of Excitons (Intersoienoe, London, 1966).

${ }^{18}$ J. L. Hunter and H. D. Dardy, J. Acoust. Soo. Am. 36, 1914 (1964); 8. S. Yun and R. T. Beyer, J. Chem. Phys, 40, 2538 (1974).

${ }^{19}$ L. Liebermann, Phys. Rev. 113, 1052 (1959); in Physical Acoustics, edited by E. Mason (Academic, Now York, 1973), Vol. IV; H. G. Denielmeyer, Acustica 17, 109 (1966). 